\title{
Zur Biologie von Gonatopus pilosus Thoms.
}

Ein hymenopterologischer Beitrag von Josef Mik. (Hierzu Taf. III.)

Bei Geleğenheit einer Excursion im vorigen Sommer machte mich mein Freund Paul Löw auf die beutelförmigen Auswüchse aufmerksam, welche man nicht selten an Cicadellinen findet. Er habe aber trotz mancher Zuchtversuche nicht ermitteln können, welcher Parasit diese Auswüchse verursache; auch war ihm nicht bekannt, dass jemand Anderer hierüber Aufschluss gegeben hätte. ${ }^{1}$ ) Da wir uns erinnerten, dass Boheman ein Dipteron ${ }^{2}$ ) aus einer Cicadine gezogen hatte, so beschloss ich. der Sache meine volle Aufmerksamkeit zuzuwenden.

1) Auf dem Abdomen exotischer Fulgoriden finden sich, ebenfalls in eigenen Säcken, Schmetterlinge als Parasiten; man vgl. Transact. Entom. Soc. London 1876. pag. 519. Pl. VII. und 1877, pag. 433, Pl. Xr. - Erst nachdem mir die Zucht aus den oben erwähnten Ciøadinen-Auswüchsen gelungen und vorstehender Artikel fertiggestellt war, theilte mir Herr P. Löw aus seinem reichen Schatze gesammelten litterarischen Materiales mit, worauf er eben erst in letzterer Zeit gekommen sei, dass Perris bereits in seinen "Nouvelles excursions dans les grandes Landes" über die genannten Auswüchse und deren Veranlasser Aufklärung gegeben habe. Da diese Mittheilung meinen Artikel weiter nicht alterirt, als nur die Annahme richtig stellt, es sei über diese Auswüchse noch nichts bekannt gemacht worden, so änderte ich an dem Artikel selbst nichts, sehe mich aber veranlasst, Perris' Angaben, welche mir Herr P. Löw freundlichst überliess, hier zu reproduciren, weil sie nicht leicht Jedermann zugänglich sind. Perris schreibt in Ann. soc. Linn. Lyon, sèr. 2, T. IV. 1857, pag. 172-173, Folgendes: „Nous remarquâmes sur quelques individus de l'Athysamus maritimus m. un corps sphérique brun, dont la partie antérieure était engagée dans interséction de deux segments abdominaux, comme on le voit pour les Rhipiptères parasites des Hymenoptères. C'était pour nous indice d'un parasitisme intéressant et pour éclairer la question, j'enprissonai trois Athysamus ainsi attaqués dans autant de petits tubes. Quatre jours après, je montrai à mon ami Dufour une petite larve, qui filait de la soie au fond du tube et s'enreloppait d'un cocon. Nous en fûmes surpris l'un et l'autre, parce que nous nous attendions à un Rhipiptère quelconque, et nous n'en fûmes que plus intrigués. L'Hémiptère était mort; le corps globuleux qu'il portait était fendu et ouvert comme une coquille bivalve, et dans son intérieur était un autre fourreau membraneux, de même couleur, ellipsoidal et déchiré à l'un des bouts. La larve continua à filer pendant près de deux jours; elle fit, sans en souffrir, le voyage de Biscarrosse à Montede-Marsan, et peu de temps après mon retour, elle me donna le Gonatopus pedestris Dalm. Cet Hymenoptère est-il le vrai parasite de l'Athysanus, ou bien le parasite de son parasite? Je pencherais pour eette dernière hypothèse, à cause des deux enveloppes très distinctes que présente le globule noir."

2) Es war Pipunculus fuscipes Fall., welcher als Larve in dem Hinterleibe von Cicadula virescens Fall. (id est Thamnotettix sulphurella Zett.) 
In der zweiten Hälfte des Monates Juli vorigen Jahres streifte ich auf einer feuchten Wiese nächst $\mathrm{Hammern}$ bei Freistadt in Oberösterreich einige Cicadellinen-Nymphen mit den genannten Auswüchsen. Fig. 1-3 (Taf. III) zeigt eine solche Nymphe von oben, von unten und von der Seite, und man kann aus der Grössenangabe des Thieres in Fig. 3 die Kleinheit des Beutels in diesem Stadium ermessen. Seine Färbung ist schwärzlich, etwas in's Neutralblaue gehend: seine Hülle ist ziemlich stark chitinisirt, glatt, auf den Seiten etwas abgeplattet und daselbst glänzend; längs des ganzen Rückens verläuft eine eingeschobene Schiene, welche matt ist und Spuren einer Segmentirung zeigt; an der Bauchseite des Beutels befindet sich ein Längseindruck. Ich fand 3 Nymphen mit derartigen Ausw üchsen behaftet; jedesmal kam der Beutel an der rechten Seite des Hinterleibes des Wirthes hinter dem zweiten Segmente hervor, doch vermuthe ich darin keine Regel. Der Auswuchs verursacht eine geringe Asymetrie in der Ausbildung der vorhergehenden Ringe und des Metathorax, wie aus Fig. 1 ersichtlich ist. Bei einem Exemplare zeigte sich statt der erwähnten Rückenplatte am Beutel nur eine Rückennaht, in welcher die beiden Seitenklappen zusammenstiessen. Ich halte dafür, dass die Hülle des Beutels organisch mit der darin lebenden Larve verbunden und als ihre äussere Haut zu betrachten sei; das Wachsthum dieser Haut wird vorzüglich dadurch erfolgen, dass die Rückenplatte sich einschiebt und immer breiter wird. Ich konnte die zarten Nymphen der Cicadinen nicht länger als ein paar Stunden lebend erhalten, und gab daher meine Hoffnung auf ein günstiges Zuchtresultat auf. Das wenige Materiale, welches mir zu Gebote stand (2 Thiere sind gänzlich eingetrocknet), erlaubte mir keine eingehenden Untersuchungen bezüglich der Larven in diesem Stadium; sie bleiben einem Anderen überlassen. So viel muss ich aber erwähnen, dass die eine Larve. welche ich untersucht habe, mit der Hülle des Beutels im innigen Zusammenhange stand, dass man bereits die Anfänge der Oberkiefer und der Taster wahrnahm, dass ferner der Fettkörper sich vollgepfropft von ziemlich grossen, prismatischen, farblosen Krystallkörperchen zeigte. Dieselben dürften harnsauere

schmarntzte; die Verpuppung erfolgte in der Erde. Conf. Boh. Oefv. Vet. Akad. Förh. 1854 , p. 302-305. Tab. V. In Schiner's Fauna austr. I. p. 245 findet sich ein lapsus calami: es heisst daselbst, dass Pipunculus im Leibe einer Cicindela gefunden wurde. Herr Paul Löw traf eine Pipunculus-Larve im Hinterleibe von Grypotes puncticollis H. S. 
Salze sein, dem gerade rhombischen Systeme angehören und waren 0.3 bis $0.35 \mathrm{~mm}$ lang und 0.05 bis $0.2 \mathrm{~mm}$ breit. Die Hülle der Seitentheile des Beutels zeigt abwechselnd hellere und dunklere, schmale, zickzackähnlich verlaufende Streifen unter dem Mikroskope; ob diese eine Folge ron Structurverhältnissen oder nur Farbenstreifen sind, konnte ich bei dem wenigen vorhandenen Materiale nicht eruiren. An der gleichmässig und heller tingirten Rückenplatte zeigen sich zwei Reihen von kurzen, hohlen, haarähnlichen Fortsätzen; ob sie Aus- oder Einstülpungen der Chitinhülle des Beutels sind, ob sie daher äusserlich auftreten oder in den Beutel hineinragen, bleibt ebenso fraglich, wie die Function dieser Gebilde. Ich zählte deren in jeder Reihe sieben; ebenso glaubte ich ähnliche Fortsätze an den Seitentheilen des Beutels bemerkt zu haben, wo sie gleichfalls in je eine bogenförmige Reihe vertheilt waren. Offenbar entsprechen sie der auf der Rückenplatte angedeuteten Segmentirung.

Am 1. September gelang es mir erst wieder, trotz fleissigen Suchens an derselben Stelle, von welcher die Cicadinen-Nymphen stammten, zwei mit Beuteln versehene Individuen desselben Thieres, ${ }^{3}$ ) aber im ausgewachsenen Zustande einzufangen. Die Auswüchse waren hirsekorngross, also bedeutend grösser als an den Nymphen, sonst aber ihrer ganzen Bildung nach nicht verändert. Die Träger hüpften munter herum - und unterschieden sich bei oberflächlicher Betrachtung von normalen Exemplaren nur dadurch, dass sie das rechte Flügelpaar etwas gelüftet trugen. Ich zwingerte sie in ein Zuckerglas ein, auf dessen Boden sich gereinigte Erde befand, und stellte jene Pflanzen hinein, welche am Fangplatze vorkamen: Glyceria fluitans, Parnassia pal., Succisa pratens., Ranunculus acris und einige Carices. Die Cicadinen sogen beide alsbald am liebsten an den Blättern von Glyceria, verschmähten aber auch nicht die Stengel von Parnassia. Am 3. September setzte sich Nachmittags eine der beiden Cicadinen zum Saugen an ein Blatt fest und verharrte in dieser Stellung unverrückt noch am Abend; am nächsten Morgen sah ich sie noch in derselben Stellung, doch völlig durchsichtig, und der Beutel war an Rücken längs der Mittellinie der quergestreiften Schiene aufgesprungen, klaffte muschelartig und war leer. Ich fand im Glase zwischen den Blüthen

$\left.{ }^{3}\right)$ Herr Paul Löw determinirte das Thier als Deltocephalus xanthoneurus Fieb. 
eines Succisa-Köpfchens eine lebhaft umherkriechende, feiste Larve, welche ohne $Z$ weifel dem Beutel entschlüpft war, da am nächsten Tage auch aus der anderen Cicadine eine zweite Larve von derselben Beschaffenheit ausgefallen war. Letztere benützte ich zur Untersuchung, so dass nur mehr das einzelne erste Stück zum Zuchtrersuche übrig geblieben war.

Bei solchem spärlichen Materiale von Larven auch in diesem Stadium bleiben selbstverständlich eine Menge Fragen offen, deren Beantwortung andere Biologen übernehmen mögen; doch kann man sich die Entwicklung der Larve bis zu jenem Stadium, wo sie den Beutel verlässt, in Folge meines Zuchtversuches dadurch sichern, dass man nur ausgewachsene, mit dem Schmarotzer behaftete Cicadinen einträgt und sie in oben geschilderter Weise ernährt. Wann sich die Larve von der Beutelhülle ablöst, wie sie sich ernährt. sind eben solche offene Fragen. Ich denke mir das Ablösen von der Beutelhaut als einen einfachen Häutungsprocess. ${ }^{4}$ ) Das schnell erfolgende Durchsichtigwerden des Wirthes setzt voraus, dass der Schmarotzer in den letzten Stunden einen besonderen Heisshunger entwickeln muss, zugleich lässt es aber die nahe liegende Vermuthung, ob hier nicht ein Schmarotzer im Schmarotzer lebte, nicht recht aufkommen; denn wäre dies der Fall, so hätte sich der letzte Schmarotzer wohl mit dem Aufzehren des ersten begnügt. $O b$ die Larve tief in den Leib des Wirthes hineinragt oder einzudringen vermag, ob sie frisst oder saugt, zu welchem Zwecke die sehr grossen Oberkiefer dienen; dies alles musste unentschieden bleiben.

Die Larve ist bei $4 \mathrm{~mm}$ lang, $1 \mathrm{~mm}$ breit, walzenförmig, hinten völlig stumpf, das Vorderende ist etwas schmäler, kann aber durch Vorstrecken, was namentlich beim Kriechen geschieht, sehr stark zugespitzt werden, wobei sich die Totallänge nicht unerheblich vergrössert. Ihre Farbe ist gelblichweiss; an den Seitenrändern befindet sich je ein schmaler glasheller Wulst (Fig. 4), welcher den Rücken vom Bauche trennt; ersterer ist convexer als letzterer. Die Segmentirung ist sehr undeutlich; ich zählte 13 Segmente nebst dem Kopfe. An den Seiten jedes Segmentes steht eine starke,

4) Wenn Perris, wie wir oben gelesen, noch von einer zweiten Hülle innerhalb des aufgesprungenen Beutels spricht, so ist noch kein Grund vorhanden, dass man es hier mit einem Kuckucks-Schmarotzer zu thun habe; es ist dies wahrscheinlich der Rest eines zweiten Häutungsprocesses, der noch innerhalb der bereits von der Larve abgestossenen Beutelhülle vor sich gegangen ist. 
gerade, weisse Borste, am Rücken trägt jedes Segment zwei kürzere solche Borsten (Fig. 5). Am Kopfe fallen die sehr grossen, hornigen, gelbbraunen Oberkiefer auf; die Laden sind an der Spitze schwarz und besitzen am Innenrande, nahe der Basis, einen Ausschnitt. Das Basalstück zeigt nach innen einen eckigen Vorsprung (Fig. 6). Die schildförmige Oberlippe (labrum) ist sehr gross, am Vorderrande gewimpert, von der Farbe des übrigen Larvenkörpers. Die Kopfkapsel ist oberseits graulich, glänzend, mit einer Mittelfurche versehen; am Seitenrande ist sie gewulstet, am Clypeus vorn mit einigen schwarzen Haaren besetzt; die Augen sind klein. einfach, stark glänzend, schwarz und stehen neben dem Wulste, dessen Furche sich hakenförmig über sie hereinbiegt; neben dieser Furche befindet sich je ein längeres schwarzes Haar (Fig. 5). Die Unterlippe (labium) ist quergestellt, jederseits zweimal seicht eingebuchtet; sie ist weisslich, schwärzlich gerandet, fein eingestochen punktirt, und zeigt am Hinterrande einen quergestellten, schwärzlichen Pigmentfleck. Die Unterkiefer sind klein aber stark, weisslich und schliessen eine Spalte ein; vielleicht bilden sie ein Saugorgan; ihre Taster stehen auf einem dicken. lichtgelben Basalgliede, welches mit einzelnen längeren, weissen Haaren besetzt ist, sind stielartig, ockergelb und tragen an der Spitze einen ebenso gefärbten, kurzen, spitzen, excentrisch angesetzten Griffel (Fig. 6).

Die Bewegung der Larve ist sehr lebhaft, wurmförmig; beim Kriechen, welches merkwürdigerweise stets auf dem Rücken erfolgt, zieht sie die hinterste Körperpartie zusammen und erscheint hier auffallend verdickt; diese Verdickung schreitet dann rasch gegen vorwärts zu fort, während der hinter der Verdickung gelegene Theil sofort sein normales Volumen annimmt. Die hierbei erzeugte Formveränderung der Larve ist insbesondere deshalb so auffallend, weil immer nur eine sehr kleine Partie des Körpers sich rasch nacheinander und sehr stark verdickt. Während des Kriechens schlägt die Larve den Kopf, ihn bald links, bald rechts erhebend, auf sich zurück und spinnt dabei fortwährend feine Fäden um sich, mit welchen sie sich wohl an der Unterlage befestigt; die steifen Borsten am Rücken 'werden zum Vorwärtsschieben benützt.

So bewegte sich die Larve unstät und scheinbar spürend und suchend auf der Erde in ihrem Zwinger bis zum 7. September, ohne eine Nahrung zu sich zu nehmen. An diesem Tage brachte ich sie in eine kleine Eprouvette, in welcher am nächsten Morgen an der Seitenwand bereits ein schütteres, schmal ellipsoidisches, 
weisses Gespinnste von $4.7 \mathrm{~mm}$ Länge wahrzunehmen war, welches nur eine Decke über der Larve bildete und mit den Rändern an die Glaswand befestigt war; rings um das Gespinnst waren einige verworrene Fäden früher an das Glas angelegt worden. Am 9. September Morgens war bereits eine zweite dichtere Coconschichte von Biscuitform im Innern des ersten Gespinnstes (Fig. 7) fertig geworden, welche auch die Glaswand überzog. Die Larve spann noch am 13. September, an welchem Tage ich nach Wien abreiste. Doch blieb der Cocon an der Seite der Glaswand so schütter, dass man die Larve und später auch die Puppe sammt ihren Bewegungen ganz gut wahrnehmen konnte.

In Wien brachte ich das Gläschen in einer geschlossenen Schachtel zwischen die Fenster eines ungeheizten Raumes, ohne es den ganzen Winter hindurch anzufeuchten. Ich überliess Alles dem Zufalle. Am 16. April 1882 war die Larve im Cocon an ihrem schwarzen Pigmentflecke der Unterlippe noch zu erkennen, auch gab sie noch Lebenszeichen von sich, doch erfüllte sie um diese Zeit an ihrem hinteren Ende nicht mehr den ganzen Cocon, sie hatte sich etwas zusammengezogen. - Am 10. Mai konnte ich keine Bewegung mehr wahrnehmen, gegen den Hintertheil des Körpers zu zeigte sich eine unregelmässige dunkle Masse. Ich gab von nun an alle acht Tage ein Stückchen angefeuchtetes Flusspapier in das mit Tüll überzogene Gläschen. $\mathrm{Am}$ 12. Mai war die Larve wieder durch den ganzen Cocon gestreckt, der dunkle Inhalt war etwas kleiner geworden und mehr nach vorwärts gewandert; am nunmehr spitz gewordenen Hinterende zeigte sich eine kleine schwarzbraune Exuvienmasse. Ich war nun sicher, dass sich die Larve gehäutet hatte und ihrer Verpuppung entgegengehe. Der Fleck, welcher den dunkeln Inhalt markirte, rückte von Tag zu Tag weiter nach vorn und vergrösserte sich. Am 1. Juni war die ganze Puppe dunkelbraun, bis auf den Raum zwischen den grossen schwarzen Augen. Man konnte den grossen abgeschnürten Kopf, den Thorax, die Fühler und die Beine der Mumienpuppe wahrnehmen. Am 8. Juni schlüpfte die Imago vor meinen Augen aus, nachdem zuvor der Cocon am Kopfende nach und nach durchnagt worden war.

Das Thier erwies sich als ein Weibchen von Gonatopus pilosus Thoms. (Köngl. Vetensk. Akad. Stockholm 1860, p. 180, Nr. 2.) Für die richtige Determinirung desselben bürgt die Autorität des bekannten Hymenopterologen Dr. Gust. Mayr. Ich habe dieses merk- 
würdige Thier in Fig. 8-10 abgebildet und möchte zum Schlusse noch Folgendes erwähnen.

Der lange Zeitraum zwischen dem Ausfallen der Larve aus dem Beutel und ibrer Verspinnung, das unruhige und fortwährende Herumkriechen der Larve während dieser Zeit und der Umstand, dass sie in dem Cocon völlig trocken gehalten wurde, während sie von den Cicadinen beim Verlassen derselben im Freien gewiss an einem feuchten Orte abgesetzt wird, lässt schliessen, dass die Larve mitunter auch weiter entfernte trockene Orte, Baumrinden ete. zum Zwecke ihrer Verpuppung aufzusuchen in den Stand gesetzt ist. Die merkwürdige Bildung der Vorderfüsse, welche nur dem Weibchen zukommt, lässt vermuthen, dass diese und verwandte Arten mit solchen Füssen dieselben als Klammerorgane bei dem Geschäfte des Eierablegens den springenden Cicadinen gegenüber wohl zur Verwendung bringen werden. Sonst fasst man die Füsse der GonatopusWeibchen als Raubfüsse auf. An meinem Thiere konnte ich auch beobachten, dass es beim Gehen die Klauen der Vorderbeine nicht benützt, sondern nur den stärker entwickelten Haftlappen. Aus Fig. 10 ersieht man, dass die eine Klaue auffallend verlängert, stark und säbelförmig, die andere ebenfalls sehr gross, doch zu einem eigenthümlichen, fast löffelförmigen Organe umgewandelt ist; letztere ist überdies noch gegen den Tarsus zurückgeschlagen, kann jedoch von demselben nicht weiter abgehoben werden, als es die Zeichnung zeigt, während die säbelförmige Klaue frei beweglich ist. Beim Gehen nun wird diese an die umgewandelte Klaue völlig angelegt, so dass beide Klauen einer geschlossenen Scheere gleichen; sie können so fest zusammengehalten werden, dass es einer ziemlichen Kraft bedarf, um sie zu öffnen.

Erklärung der Tafel III. Fig. 1. Nymphe von Deltocephalus xanthoneurus Fieb. - Fig. 2. Der Hinterleib derselben von unten. - Fig. 3. Dieselbe von der Seite. - Fig. 4. Die Larve von Gonatopus pilosus Thoms., von der Bauchseite gesehen. - Fig. 5. Das Vorderende derselben von oben, Fig. 6 von unten, stärker vergrössert. - Fig. 7. Der Cocon dieses Thieres von der Unterseite. - Fig. 8. Weibchen von Gonatopus pilosus Thoms. in der Aufsicht, Fig. 9 im Profile. - Fig. 10. Das rechte Vorderbein der Imago, stärker vergrössert; $\mathrm{e}=\operatorname{cox} a, \mathrm{tr}=$ trochanter, $\mathrm{f}=$ femur, $\mathrm{t}=$ tibia. 

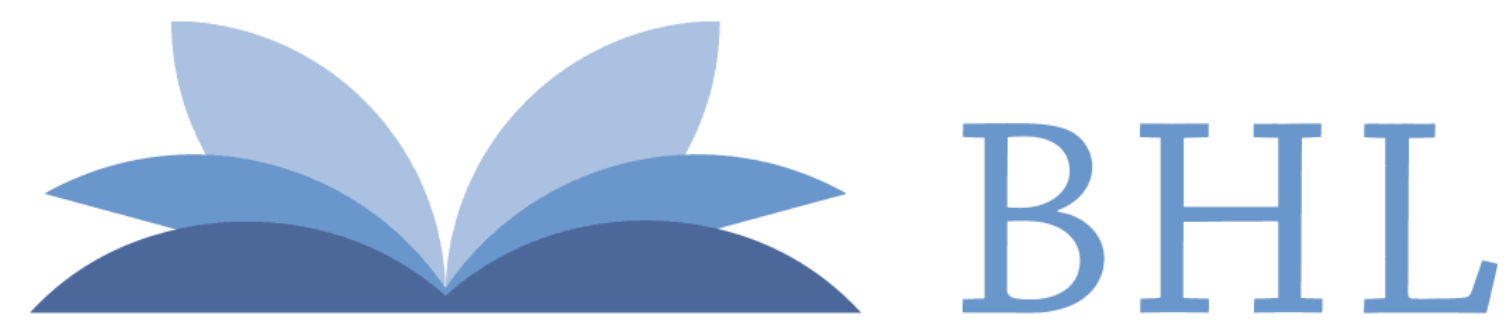

\section{Biodiversity Heritage Library}

Mik, Josef. 1882. "Zur Biologie von Gonatopus pilosus THOMS. 1 Tafel." Wiener entomologische Zeitung 1, 215-221. https://doi.org/10.5962/bhl.part.11161.

View This Item Online: https://www.biodiversitylibrary.org/item/42649

DOI: https://doi.org/10.5962/bhl.part.11161

Permalink: https://www.biodiversitylibrary.org/partpdf/11161

\section{Holding Institution}

Smithsonian Libraries

\section{Sponsored by}

Smithsonian

\section{Copyright \& Reuse}

Copyright Status: NOT_IN_COPYRIGHT

This document was created from content at the Biodiversity Heritage Library, the world's largest open access digital library for biodiversity literature and archives. Visit BHL at https://www.biodiversitylibrary.org. 